\title{
OPERATOR-WEIGHTED COMPOSITION OPERATORS ON VECTOR-VALUED BLOCH SPACES
}

\author{
Mostafa HASSANLOU
}

Abstract. Let $\varphi$ be an analytic self-map of $\mathbb{D}$ and $\psi$ be an analytic operator-valued function on $\mathbb{D}$. Then the operator-weighted composition operator $W_{\psi, \varphi}$ is defined by

$$
\left(W_{\psi, \varphi} f\right)(z)=\psi(z) f(\varphi(z)), \quad z \in \mathbb{D},
$$

where $f$ is an analytic function $\mathbb{D} \rightarrow X, X$ is any complex Banach space. In this paper by considering $W_{\psi, \varphi}$ on vector-valued Bloch spaces, some qualitative properties of these operators will be characterized.

Mathematics subject classification (2010): 47B33, 46E15, 46E40, 47B07.

Keywords and phrases: Analytic functions, Banach space, compactness, composition operator, weak compactness, vector-valued function.

\section{REFERENCES}

[1] F. Albiac And N. J. Kalton, Topics in Banach Space Theory, Springer, 2006.

[2] J. BONET, P. DOMANSKI AND M. LiNDSTROM, Weakly compact composition operators on analytic vector-valued function spaces, Ann. Acad. Sci. Fenn. Math. 26 (2001), 233-248.

[3] J. Bonet, M. C. Gómez-Collado, D. Jornet and E. Wolf, Operator-weighted composition operators between weighted spaces of vector-valued analytic functions, Ann. Acad. Sci. Fenn. Math. 37 (2012), 319-338.

[4] C. C. Cowen AND B. D. Maccluer, Composition operators on spaces of analytic functions, Studies in Advanced Mathematics, CRC Press, Boca Raton, Fla, USA, 1995.

[5] J. Diestel, H. Jarchow and A. Tonge, Absolutely Summing Operators, Cambridge Univ. Press, Cambridge 1995.

[6] M. Hassanlou, J. Laitila AND H. VAeZI, Weighted composition operators between weak spaces of vector-valued analytic functions, Analysis, 37 (1) (2017), 39-45.

[7] E. Hille AND R. S. Phillips, Functional Analysis and Semigroups, Amer. Math. Soc. Providence, R. I. 1957.

[8] J. LAitila, Weakly compact composition operators on vector-valued BMOA, J. Math. Anal. Appl. 308 (2005), 730-745.

[9] J. LAitila, Composition operators and vector-valued BMOA, Integr. Equ. Oper. Theory, 58 (2007), 487-502.

[10] J. Laitila, P. J. Nieminen, E. Saksman And H.-O. Tylli, Compact and weakly compact composition operators on BMOA, Complex Analysis and Operator Theory 7 (2013), 163-181.

[11] J. LAITILA AND H.-O. TYLLI, Operator-weighted composition operators on vector-valued analytic function spaces, Illinois J. Math. 53 (2009), 1019-1032.

[12] J. Laitila, H.-O. TYLli, AND M. WANG, Composition operators from weak to strong spaces of vector-valued analytic functions, J. Operator Theory 62 (2009), 281-295.

[13] P. Liu, E. Saksman And H.-O. Tylli, Small composition operators on analytic vector-valued function spaces, Pacific J. Math. 184 (1998), 295-309.

[14] J. S. MANHAS, Weighted composition operators on weighted spaces of vector-valued analytic functions, J. Korean Math. Soc. 45 (2008), 1203-1220.

[15] M. NowAK, On Bloch space in the unit ball of $\mathbb{C}^{n}$, Ann. Acad. Sci. Fenn. 23 (1998), 461-473. 
[16] S. Ohno, K. Stroethoff And R. Zhao, Weighted composition operators between Bloch-type spaces, Rocky Mountain J. Math. 33 (1) (2003), 191-215.

[17] J.H. ShapIRO, Composition operator and classical function theory, Springer-Verlag, New York, 1993.

[18] M. WANG, Weighted composition operators between Dirichlet spaces, Acta Math. Sci. 2011, 31B (2): 641-651.

[19] M. WANG, Riemann-Stieltjes operators between vector-valued weighted Bloch spaces, J. Ineq. Appl., (2008), doi: 10.1155/2008/348208.

[20] K. ZHU, Spaces of holomorphic functions in the unit ball, Springer, New York, 2005. 